\title{
Optimization on Vehicle Routing Problem based on Immune Clonal Selection Algorithm
}

\author{
Haihua $\mathrm{Li}^{\mathrm{a}}$, Zongyan $\mathrm{Xu}^{\mathrm{b}}$ and Feifei Zhou \\ Military Transportation Department, Military Transportation University, Tianjin, China \\ ahh.i@qq.com, ${ }^{b} x z y 2012 t j @ 163 . c o m$
}

Keywords: immune clonal selection; vehicle routing problem; genetic algorithm

\begin{abstract}
Vehicle routing problem is a typical NP-hard problem and is difficult to get an optimum solution. Aiming at the shortages of the existing methods, this paper proposed an algorithm based on immune clonal selection to solve vehicle routing problem. In the algorithm, expressed antibody with matrix, generated the initial population of antibodies randomly, and employed the operations such as clonal selection, genetic mutation iteratively to search optimum solution in solution space. The experimental results show that the algorithm presented here can converge to the global optimum solution rapidly, overcoming such disadvantages of the genetic algorithm as slower convergent velocity and the convergence to a local optimum solution.
\end{abstract}

\section{Introduction}

Vehicle routing problem (VRP) was advanced by Dantzig and Ramser in 1959, which had always became the hot issue in the field of operations research and combinatorial optimization. In practice, many problems such as the postal delivery problem, the scheduling problems in aircraft, trains, water transport ship, and the power dispatch problem can be abstracted as the VRP. Therefore, the study for VRP has important theoretical and practical significance.

Due to VRP is an NP-hard problem, its solution will be a sharp increase in an exponential rate with the increase of the number of customers. Therefore, heuristic algorithms for solving this problem have become an important research direction. There are many methods, such as dynamic programming, saving algorithm and scanning algorithm, to solving VRP. However, these algorithms cannot effectively solve the large scale problem. In order to improve computational efficiency, the heuristic algorithms like tabu search algorithm and simulated annealing algorithm are used to solve this problem.

On the basis of describing the vehicle routing problem briefly, this paper presents an algorithm based on immune clonal selection to solve the problem, and gives a detailed description of the main part of algorithm. The computational results show that the high quality solutions to the problem can be obtained by using the proposed immune clonal selection algorithm, and the new algorithm is also efficient and robust.

\section{Mathematical Model}

Description. VRP can be described as follows. Let $m$ be the number of vehicles in the distribution center, which maximum load is $q$. There are $n$ customers with demand $g_{i}(i=1,2, \ldots, n), g_{i}<q$. It is required to arrange reasonable routes, which the total mileage is shortest. At the same time, the following constraints must be met: (1) Customers' demands of every route do not exceed the load capacity of one vehicle; (2) The total length of each route does not exceed the maximum driving distance of one vehicle; (3) Each customer's demand must be met and each customer visits only by one vehicle.

Model. Let 0 be the distribution center, and $i(i=1,2, \ldots, n)$ be the customer node. Let $k(k=1,2, \ldots, m)$ be the vehicle in the distribution center. And let $c_{i j}$ be the distance between customer $i$ and customer $j$. Define the variables as follows: 


$$
\begin{aligned}
x_{i j k} & =\left\{\begin{array}{l}
1, \text { vehicle } k \text { travels from customer } i \text { to } j \\
0, \text { otherwise }
\end{array}\right. \\
y_{i k} & =\left\{\begin{array}{l}
1, \text { customer } i \text { is served by vehicle } k \\
0, \text { otherwise }
\end{array}\right.
\end{aligned}
$$

Then the VRP model can be formulated as follows:

$$
\min \quad z=\sum_{i=0}^{n} \sum_{j=0}^{n} \sum_{k=1}^{M} c_{i j} \cdot x_{i j k},
$$

Subject to:

$$
\begin{gathered}
\sum_{i=1}^{n} q_{i} \cdot y_{i k} \leq q \quad, k=1,2, \ldots, m, \\
\sum_{k=1}^{m} y_{i k}=\left\{\begin{array}{ll}
m & i=0 \\
1 & i=1,2, \ldots, n
\end{array},\right. \\
\sum_{i=0}^{n} x_{i j k}=y_{j k}, \\
\sum_{j=0}^{n} x_{i j k}=y_{i k},
\end{gathered}
$$

Where, constraints (4) represent the total customers' demand on each route is not greater than the load capacity of one vehicle; constraints (5) denote that the distribution tasks are completed by $m$ vehicles, and each customer visits only by a vehicle; constraints (6) and (7) ensure that the vehicle reaches the customer and must leave it.

\section{The Immune Clonal Selection Algorithm}

The clonal selection is an importation doctrine of the biological immune theory. Many people like L.N.DeCastro proposed different clonal selection algorithms by simulated clonal selection mechanism from different angles. Among them, the algorithm using clonal selection operator is called the immune clonal selection algorithm (ICSA). There are advantages of ICSA to increase the diversity of the population. The basic steps of ICSA can be described as followings:

Step1: Let $k=0$, operate initialization for antibody population, and calculate the affinity of the initial population.

Step2: According to the affinity and the size of antibody population, implement the clonal operator, the immune genetic operator, and the clonal selection operator to obtain the new antibody population.

Step3: Calculate the affinities of antibodies in the new antibody population.

Step4: Let $k=k+1$. If it achieves the termination condition, then terminate; otherwise return to Step2.

Antibody Initialization. In ICSA, the antibody is corresponding to the candidate solution in practical problems. The collections of antibodies are known as the antibody population. In practice, the antibody is denoted as the encoding. There are many encoding forms such as binary and decimal, and many antibody' expressions such as linear notation and matrix notation.

In this article, we adopted the binary encoding and the matrix notation. Each candidate solution in the VRP with size $m \times n$ could be represented as an $m \times n$ matrix. For example, there are three distribution centers and four customers in VRP, then the antibody could be expressed as a $3 \times 4$ matrix, namely 


$$
\operatorname{Aag}_{k}=\left(\begin{array}{ccc}
a_{11} & \cdots & a_{14} \\
\vdots & & \vdots \\
a_{31} & \cdots & a_{34}
\end{array}\right) .
$$

where $a_{m n}$ represents the route from distribution center $m$ to customer $n$. If there is a route between them, then $a_{m n}=1$; otherwise $a_{m n}=0$.

Clonal Operation. The clonal operation is to replicate the outstanding individuals in the population by simulated learning and memory. The function of clonal size is defined as

$$
N_{c}=\sum_{i=1}^{N} \operatorname{round}(\beta \times N / i)
$$

Where $N_{c}$ is the size of antibody population; $\beta$ is clonal coefficient, which is used to control the size of clone; round is rounding function. When implementing clone, according to affinity, we can order antibodies in descending, and $\operatorname{round}(\beta \times N / i)$ antibodies would be cloned by the $i$-th antibody. So the greater size of clone would be obtained by antibody with higher affinity, which ensures that the good genes in the high-affinity antibodies have reserved.

Immune Gene Operation. The clonal selection algorithm is emphasis on the mutation. Mutation is an input parameter to determining the probability that a given antibody is mutated in each iteration. According to the characteristics of VRP and the selected encoding, the mutation strategy in this paper was as follows: randomly selected a certain rows and columns of the original matrix to form a sub-matrix, then inserted it back to the original matrix after mutation.

Affinity Calculation: Evaluation and Selection. In artificial immune system, the affinity of the antibody and antigen is generally corresponding to the objective function value of candidate solution. If the affinity is greater, then the antibody is more excellent. Here affinity could be adopted the objective function of VRP, that is

$$
f(x)=\sum_{i=0}^{n} \sum_{j=0}^{n} \sum_{k=1}^{M} c_{i j} \cdot x_{i j k}
$$

For the antibody population with size $N$, select $m(m \leq N)$ antibodies with larger affinities into excellent unit, and put the remaining $N-m$ antibodies into the bad unit, then implement clonal operation only for the antibodies in excellent unit to avoid greedy searching when all antibodies are selected to clone.

\section{Algorithm Steps}

Step1: Randomly generate initial antibody population, i.e., $\operatorname{Aag}_{i}=\left[\operatorname{Aag}_{i k}\right](i=0,1, \ldots, N ; k=1,2, \ldots, N)$.

Step2: Calculate the affinity of antibody and antigen by (10).

Step3: Express the antibody population as $\mathrm{Aag}_{i}=\mathrm{Aag}_{\text {i-excellent }} \cup \mathrm{Aag}_{\text {i-bad }}$ in accordance with the affinity obtained from Step2.

Step4: Make clonal operation for $\mathrm{Aag}_{\text {i-excellent }}$ which size is determined by (9).

Step5: Operate the mutation for the cloned antibodies. Other antibodies which do not enter the memory unit are operated immune gene operation(mutation) in accordance with a certain probability to generate $\operatorname{Aag}_{i}^{\prime}, \operatorname{Aag}_{i}^{\prime \prime}, \operatorname{Aag}_{i}^{\prime \prime \prime}, \ldots$

Step6: Evaluation and selection. Calculate affinity and get rid of the antibodies with smaller affinities to keep the population size $N$, then get into the next generation $\operatorname{Aag}_{i+1}=\left[\operatorname{Aag}_{i}^{\prime}, \operatorname{Aag}_{i}^{\prime \prime}, \operatorname{Aag}_{i}^{\prime \prime \prime}, \ldots\right]$.

Step7: If it met the termination condition or got the optimal solution, then terminated; otherwise, returned to Step3. 


\section{Computational Experiment}

One test problem was randomly constructed with 5 vehicles, which maximum load capacity was $7 \mathrm{t}$. The maximum travel distance of a vehicle was $50 \mathrm{~km}$. There were 20 customers. Randomly generated the distribution center' coordinates $(14.5,13.0)$, other data were shown in table 1. For simplicity, the distance was using the straight-line distance, which can be calculated in accordance with the coordinates of customers and distribution center.

Table1.Source Data

\begin{tabular}{ccccccccccc}
\hline customer & 1 & 2 & 3 & 4 & 5 & 6 & 7 & 8 & 9 & 10 \\
$x$ & 18.7 & 3.9 & 9.6 & 6.8 & 12.8 & 8.6 & 12.5 & 13.8 & 6.4 & 13.3 \\
$y$ & 15.4 & 10.6 & 14.8 & 16.9 & 8.6 & 8.4 & 2.7 & 5.4 & 5.6 & 15.6 \\
$q$ & 1.5 & 0.3 & 0.5 & 0.9 & 0.1 & 0.6 & 1.2 & 0.4 & 0.7 & 1.4 \\
\hline \hline customer & 11 & 12 & 13 & 14 & 15 & 1 & 17 & 18 & 19 & 20 \\
$x$ & 11.9 & 18.4 & 14.8 & 0.3 & 15.4 & 8.8 & 10.6 & 1.8 & 17.7 & 7.4 \\
$y$ & 19.8 & 3.4 & 2.6 & 2.8 & 16.6 & 8.4 & 7.6 & 8.7 & 11.0 & 1.6 \\
$q$ & 1.5 & 0.4 & 1.3 & 1.2 & 1.1 & 0.6 & 1.7 & 1.3 & 0.1 & 0.7 \\
\hline
\end{tabular}

In the paper, the parameters were set as follows: population size 150, Aag $_{\text {i-excellent }}$ size 30, mutation probability 0.1 , maximum iterations 1000 . The sub-matrix size was selected as $3 \times 5$. Calculated the program 10 times, and got the results shown in table2.

Table2. Results

\begin{tabular}{cccccccccccc}
\hline times & 1 & 2 & 3 & 4 & 5 & 6 & 7 & 8 & 9 & 10 & $\begin{array}{c}\text { Average } \\
\text { value }\end{array}$ \\
mileages & 107.8 & 106.7 & 103.2 & 110.9 & 104.4 & 109.1 & 105.5 & 103.5 & 108.8 & 105.9 & 106.6 \\
vehicles & 4 & 4 & 3 & 4 & 3 & 4 & 4 & 3 & 4 & 3 & 3.6 \\
$\begin{array}{c}\text { Computing } \\
\text { time }\end{array}$ & 5.2 & 5.3 & 5.0 & 4.8 & 5.4 & 4.7 & 5.1 & 4.8 & 5.1 & 5.2 & 5.1 \\
\hline
\end{tabular}

It can be seen from table 2 that high quality solutions were obtained in 10 operations, which the average value of solutions was $106.6 \mathrm{~km}$, and the average value of used vehicles was 3.6. The highest quality solution was got in third time, which the total mileage was $103.2 \mathrm{~km}$, corresponding to three routes: route one: $0-5-17-14-18-2-4-3-0$, route two: $0-13-12-7-20-9-10-16-0$, and route three: 0-8-10-11-15-1-19-0.

Table 2 showed that the obtained results were stable in 10 times calculation, which the different value between the worst solution and the best solution was $5.4 \%$. At the same time, the average computing time in 10 operations was 5.1 s, showing the high computational efficiency.

\section{Conclusion}

This paper discussed vehicle routing problem using the immune clonal selection algorithm. The experimental results show that the designed algorithm for solving vehicle routing problem can get high quality solutions, and converge to the global optimum solutions rapidly. 


\section{References}

[1] Emmanouil E.Zachariadis, and Chris T.Kiranoudis, A Strategy for Reducing the Computational Complexity of Local Search-based Methods for the Vehicle Routing Problem, Computers \& Operations Research, 2010, 37, pp.2089-2105.

[2] Maoxiang Lang, Two-Phase Algorithm for Dynamic Distribution Vehicle Routing Problem, Journal of Transportation Systems Engineering and Information Technology, 2009, 9(4), pp.140-144.

[3] Zongyan Xu, Haihua Li, and Yilin Wang, An Improved Genetic Algorithm for Vehicle Routing Problem, Proc. IEEE International Conference on Computational and Information Sciences (ICCIS 11), IEEE Press, Oct. 2011, pp. 1132-1135, doi:10.1109/ICCIS.2011.78

[4] Zixuan Qi, Xia Chen, and Xiaopeng Tang, Optimization on fixed-charged transportation problem based on immune clonal selection algorithm, Application Research of Computers, 2009,26(7), pp.2530-2532.

[5] Jinhui Ge. Vehicle Routing Problem with Time Windows and Improved Tabu Search Algorithm[J]. Journal of Jilin University (Science Edition), 2011, 49(1), pp.105-111. 